\title{
openheart Incremental predictive value of left atrial strain and left atrial appendage function in rhythm outcome of non-valvular atrial fibrillation patients after catheter ablation
}

Xin-Xin Ma (i) , ${ }^{1}$ Aiqing Wang, ${ }^{1}$ Kaibin Lin $^{2}$

To cite: Ma X-X, Wang A, Lin K. Incremental predictive value of left atrial strain and left atrial appendage function in rhythm outcome of

non-valvular atrial fibrillation patients after catheter ablation. Open Heart 2021;8:e001635. doi:10.1136/ openhrt-2021-001635

Received 10 March 2021 Accepted 14 May 2021
Check for updates

\section{(c) Author(s) (or their} employer(s)) 2021. Re-use permitted under CC BY-NC. No commercial re-use. See rights and permissions. Published by BMJ.

${ }^{1}$ Department of Ultrasound in Medicine, Shanghai Jiao Tong University, Shanghai, China ${ }^{2}$ Department of Cardiology, Shanghai Jiao Tong University, Shanghai, China

\section{Correspondence to}

Dr Xin-Xin Ma; yuranfenglin@ outlook.com

\section{ABSTRACT}

Objective The purpose of this study was to develop a non-invasive and convenient nomogram based on speckle tracking echocardiography, left atrial appendage function and clinical factors to predict the risk of atrial fibrillation (AF) recurrence after catheter ablation.

Methods A total of 124 prospectively consecutive patients with AF treated with catheter ablation in our hospital was retrospectively analysis. Baseline echocardiographic parameters were measured by using transthoracic and transesophageal echocardiography before ablation. Multivariate analysis was performed for selecting predictors for a nomogram and internal validation and calibration were evaluated by the bootstep method. Results During the follow-up of $12 \pm 3$ months, 41 patients (33.1\%) occurred AF recurrence after catheter ablation, while 83 patients (66.9\%) had maintained sinus rhythm. Four predictors (AF type, left atrial appendage emptying flow velocity, left Atrial maximal volumes index and global longitudinal strain) with the $\mathrm{P}<0.5$ was selected into the nomogram according to multivariate findings. Internal validation by bootstrapping with 1000 resamples was determined $\mathrm{C}$-index of the nomogram for prediction AF recurrence was 0.901 , which showed optimal discrimination and calibration of the established nomogram.

Conclusions Nomogram based on echocardiography and clinical characteristics had good predictive performance for the possibility of AF recurrence, which providing practical guidance for individualised management of patients with AF after catheter ablation.

\section{INTRODUCTION}

Non-valvular atrial fibrillation (AF) is an atrial arrhythmia characterised by uncontrolled, rapid, disorganised excitation of the atria, which is associated with cardiovascular and cerebrovascular complications and morbidity, especially the risk and mortality of thromboembolic. ${ }^{12}$ It was reported that AF leads to a fivefold increase in the risk of ischaemic stroke. ${ }^{3}$ Catheter ablation,

\section{Key questions}

What is already known about this subject?

- Global longitudinal strain (GLS) of left atrial has previously been shown to predict atrial fibrillation (AF) recurrences after ablation in patients with $\mathrm{AF}$.

What does this study add?

- Left atrial appendage emptying flow velocity (LAAFV) measured by transesophageal echocardiography in patients with AF has incremental predictive value for AF recurrence after ablation.

- Nomogram based on AF type, LAAFV, Left Atrial Maximal Volumes Index and GLS had good predictive performance for the possibility of AF recurrence.

How might this impact on clinical practice?

- The findings allow for a diversified assessment of the possibility of AF recurrence.

- Nomogram in our study providing practical guidance for individualised management of patients with AF after catheter ablation.

eliminating the initiating foci of $\mathrm{AF}$, has been demonstrated to be an effective and wellestablished treatment strategy in restoring sinus rhythm and appears to be superior to antiarrhythmic therapy in symptomatic patients with drug-refractory AF. ${ }^{45}$ Although recent advances in techniques for catheter ablation, the recurrence of AF after ablation still to be observed in one third of $\mathrm{AF}$ patients and often requires repeated ablation procedures. ${ }^{6}$ Therefore, assessing the risk of individual recurrence is important for optimising the benefits of catheter ablation and selecting appropriate patients, which helps to identify those patients who are most likely to benefit from AF ablation. ${ }^{7}$ Patients with low success rate usually need to enlarge the ablation lesions according to additional left atrial (LA) substrate modification. Meanwhile, it 
also provides guidance and suggestions for the management of patients post-AF ablation.

Many prior studies have reported that AF type, LA volume index (LAVI) and plasma brain natriuretic peptide, etc, were strong predictors for AF recurrence (AFR) after catheter ablation. ${ }^{8}{ }^{9}$ Ultrasound based on speckle-tracking echocardiography (STE), as noninvasive quantification of the LA myocardial deformation properties, can identify subclinical myocardial dysfunction during the cardiac cycle, and impaired LA global longitudinal strain (GLS) are related to the degree of structural LA remodelling and fibrosis of atria in patients with AF. ${ }^{10}$ Recently, lower GLS was recognised as an independent predictor of AFR after catheter ablation. ${ }^{11} 12$ However, GLS based nomogram by comminating with LA volume and LA appendage (LAA) function have not been proposed. Thus, the present study was performed to establish a predictive model based on GLS and known predictors, and evaluate the predictive value of the proposed model.

\section{METHODS}

\section{Study population}

We prospectively enrolled 124 non-valvular AF patients who underwent their first-time ablation procedure between September 2017 and January 2020. Exclusion criteria were (1) history of moderate or greater structural heart disease, (2) active coronary artery disease or cardiomyopathy, (3) history of cardiac surgery and/or cardiac device implantation, (4) previous ablation procedure, (5) severe system chronic disease or reduced left ventricle (LV) systolic function on baseline echocardiography (LV ejection fraction, LVEF <40\%), (6) patients with atrial or ventricular arrhythmias during the echocardiographic examination, (7) patients with thrombus in LAA, (8) uninterpretable images quality and (9) unavailable of clinical follow-up data.

The baseline characteristics of patients were comprehensively assessed, including age, sex, body mass index, AF type, prevalence of the most common cardiovascular risk factors (hypertension, smoking, type 2 diabetes, dyslipidaemia), history of stroke, coronary heart disease and transient ischaemic attack/stroke, and finally current medical treatment. Plasma N-terminal pro-brain natriuretic peptide (NT-pro-BNP) concentrations were measured by a highly sensitive latex turbidimetric immunoassay before ablation. The concentrations of serum uric acid (sUA) in were quantified using enzymatic assays.

\section{Echocardiography}

\section{Standard echocardiography}

All patients underwent transthoracic echocardiography (TTE) and transesophageal echocardiography (TEE) study prior to ablation procedure by experienced sonographers, using a Philips iE33 ultrasound machines (Philips Medical Systems, Eindhoven, Netherlands) and EPIQ 7C (Philips Healthcare, Eindhoven, Netherlands), connecting limb lead ECG during TTE and TEE examination. Standard echocardiographic views were obtained and all measurements were performed according to the guidelines of American Society of Echocardiography. ${ }^{13}$ Tricuspid annular plane systolic excursion (TAPSE) was obtained via M-mode and measured. LVEF was obtained from the apical four-chamber and two-chamber views (4 CV, 2CV) by Simpson's biplane method. LA maximal volumes during diastolic phase were calculated from the apical $4 \mathrm{CV}$ and $2 \mathrm{CV}$ of the LA by the biplane method of the discs, and were indexed to body surface area, total LA Maximal Volumes Index (LAVImax).

\section{Two-dimensional STE}

Three cine loops from $4 \mathrm{CV}$ and $2 \mathrm{CV}$ acquired with at least 60 frames per second were digitised and stored for offline analysis. LA strain analysis was performed offline using commercially available software (TomTec Imaging Systems, Munich, Germany). We selected the P wave onset of the ECG as the reference point for paroxysmal AF, and the QRS wave onset of the ECG as the reference point for persistent AF, as previously proposed.$^{10} \mathrm{LA}$ endocardium boundary was traced manually in the end-systolic frame, and additional epicardial boundary were automatically marked by the software, generating a region of interest (ROI). After manually adjusting and calibrating the ROI of LA, the software automatically divided the region into six segments, and generated average time-strain curves. The software calculated LA average mean values for six segments obtained from the $4 \mathrm{CV}$ and $2 \mathrm{CV}$. We identified the LA reservoir strain as the sum values of peak positive longitudinal strain during ventricular systole and LA negative longitudinal strain during ventricular diastole, LA GLS (figure 1).

Each subject underwent TEE examination prior to catheter ablation by qualified sonographer, to observe if there were thrombus in LAA. Five-consecutive cardiac cycles were acquired in cine loop format and stored for

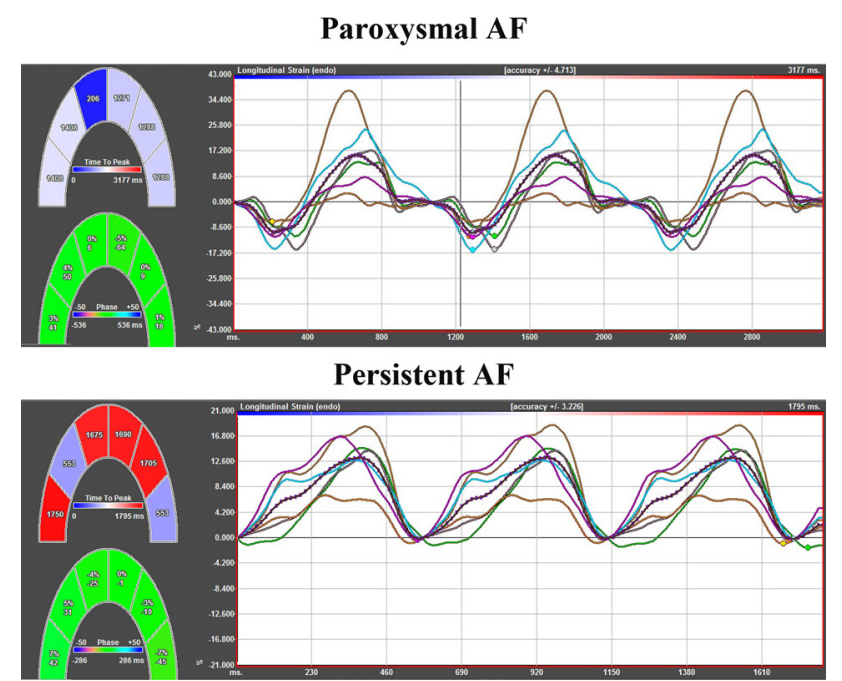

Figure 1 Screenshot of offline LA deformation analysis with 2D-speckle tracking software for paroxysmal and persistent AF. 2D, 2 dimensional; AF, atrial fibrillation; LA, left atrial. 
further analysis. LAA emptying flow velocity (LAAFV) was measured by pulse-wave Doppler with the sample volume placed at $1 \mathrm{~cm}$ away from the LAA orifice. The subjects were blindly evaluated by two experienced sonographers.

\section{Radiofrequency catheter ablation procedure}

The ablation procedure protocol at our institution was implemented as previously described. ${ }^{14}{ }^{15}$ In brief, antiarrhythmic drugs (AADs) were stopped five half-lives before ablation procedure. Pulmonary vein (PV) angiography was performed to verify the ostia and antrum of PV, CARTO mapping system (Biosense Webster, Irvine, California, USA) was constructed for building a LA threedimensional electroanatomic map and intracardiac electrogram recordings. The integration module of CARTO system was used to integrate of PV CT images with the constructed eletroanatomy to navigate the ablation catheter in real time. The endpoint of extensive circumferential PV isolation was the abolition or dissociation of PV potential or failure to induce AF. Patients with persistent $\mathrm{AF}$ received the additional ablation procedures, including linear ablation of the LA roof and isolation of superior vena cava.

\section{Follow-up}

All patients were monitored in the hospital for at least 12 hours. Oral anticoagulation was continued after the procedure and AAD therapy for 3 months. Follow-up was conventionally performed at 3,6 and 12 months after ablation. At each clinical visit, patients received a questionnaire regarding any arrhythmia-related symptoms, and followed by a 12-lead ECG and continuous 24-hour Holter monitoring. AFR was defined as a recording of $\mathrm{AF}$ or any other atrial tachyarrythmias lasting at least 30 s during electrocardiography or 24-hour Holter monitoring. However, recurrence of AF within only the first 3 months during the follow-up were defined as blanking period.

\section{Statistical analysis}

Continuous variables were expressed as mean \pm SD. Categorical data were summarised as percentages. Differences between groups were performed by using the independent-samples Student's t-test, $\chi^{2}$ statistics or Fisher's exact test if applicable. Univariate and multivariate binary logistic regression analysis were used to determine the predictors of AFR after catheter ablation, and the OR and $95 \%$ CI were calculated. Receiver operating characteristic (ROC) curve analysis were generated to assess the ability of different variables in predicting AFR, and the cutoff value was used to assess the sensitivity and specificity for prediction of AFR. The prediction model validation of nomogram was performed using bootstrap with 1000 resamples to quantify and predict future performance. Intraobserver and interobserver variability for GLS were calculated in 10 randomly chosen patients by two independent investigators, both investigators were blinded to the study background. Values of $\mathrm{p}<0.05$ were considered
Table 1 Baseline clinical, demographic and echocardiographic characteristics between the atrial fibrillation recurrence (AFR) and maintenance of sinus rhythm (MSR) groups in all subjects

\begin{tabular}{|c|c|c|c|}
\hline Variables & $\begin{array}{l}\text { AFR group } \\
(n=41)\end{array}$ & $\begin{array}{l}\text { MSR group } \\
(n=83)\end{array}$ & $P$ value \\
\hline Age (years) & $65.5 \pm 6.0$ & $62.6 \pm 7.3$ & 0.033 \\
\hline Male sex (\%) & $28(68.3 \%)$ & 47 (56.6\%) & 0.216 \\
\hline Body mass index $\left(\mathrm{kg} / \mathrm{m}^{2}\right)$ & $27.8 \pm 5.6$ & $26.5 \pm 4.9$ & 0.645 \\
\hline AF type & & & 0.114 \\
\hline Persistent AF & $26(63.4 \%)$ & $40(48.2 \%)$ & \\
\hline Paroxysmal AF & $15(36.6 \%)$ & $43(51.8 \%)$ & \\
\hline AF duration (months) & $38.7 \pm 35.5$ & $36.5 \pm 33.7$ & 0.743 \\
\hline Hypertension (\%) & $29(70.7 \%)$ & $53(63.9 \%)$ & 0.290 \\
\hline Smoking (\%) & $14(34.1 \%)$ & $32(38.6 \%)$ & 0.633 \\
\hline Type 2 diabetes (\%) & $12(29.3 \%)$ & $21(25.3 \%)$ & 0.638 \\
\hline Dyslipidaemia (\%) & $23(56.1 \%)$ & $43(51.8 \%)$ & 0.652 \\
\hline History of TIA/stroke (\%) & $11(26.8 \%)$ & $14(16.9 \%)$ & 0.144 \\
\hline History of CAD (\%) & $8(19.5 \%)$ & $12(14.5 \%)$ & 0.317 \\
\hline Antiplatelets (\%) & $10(24.4 \%)$ & $25(30.1 \%)$ & 0.328 \\
\hline ACEI/ARB (\%) & $22(53.7 \%)$ & $46(55.4 \%)$ & 0.502 \\
\hline Beta-blockers (\%) & $29(70.7 \%)$ & $64(77.1 \%)$ & 0.288 \\
\hline NT-pro-BNP (pg/mL) & $1046.0 \pm 1044.2$ & $527.8 \pm 564.9$ & 0.000 \\
\hline $\mathrm{sUA}(\mu \mathrm{mol} / \mathrm{L})$ & $345.8 \pm 106.6$ & $326.3 \pm 78.6$ & 0.254 \\
\hline TAPSE (mm) & $20 \pm 4$ & $19 \pm 4$ & 0.185 \\
\hline $\operatorname{LVMI}\left(\mathrm{g} / \mathrm{m}^{2}\right)$ & $85.8 \pm 16.6$ & $80.4 \pm 16.8$ & 0.093 \\
\hline LVEF (\%) & $59.2 \pm 7.1$ & $60.8 \pm 5.6$ & 0.161 \\
\hline LAVImax $\left(\mathrm{mL} / \mathrm{m}^{2}\right)$ & $44.1 \pm 11.4$ & $37.2 \pm 12.4$ & 0.001 \\
\hline LAAFV (cm/s) & $36.2 \pm 12.0$ & $50.3 \pm 16.9$ & 0.000 \\
\hline GLS (\%) & $19.8 \pm 4.5$ & $26.6 \pm 5.5$ & 0.000 \\
\hline
\end{tabular}

Values are expressed as mean \pm SD or frequency (\%).

ACEI/ARB, ACE inhibitor/angiotensin receptor blocker; AF, atrial fibrillation; CAD, coronary artery disease; GLS, global longitudinal strain; LAAFV, left atrial appendage emptying flow velocity; LAVImax, left atrial maximal volume index; LVEF, left ventricular ejection fraction; LVMI, left ventricular mass index; NT-pro-BNP, N-terminal pro-B-type natriuretic peptide; sUA, serum uric acid; TAPSE, tricuspid annular plane systolic excursion; TIA, transient ischaemic attack.

to be statistically significant. Statistics were conducted using SPSS V.19.0 software (SPSS) and R Studio (V.3.6.1; R studio, Boston, Massachusetts, USA).

\section{RESULT \\ Study population}

Table 1 summarises the baseline clinical and echocardiographic parameters of 128 patients that met the inclusion criteria and were followed for an average of $12 \pm 9$ months. Overall, patients were divided into two groups according to whether there was a clinical AFR, 41 patients (33.1\%) experienced AFR were defined as AFR group, while 83 patients $(66.9 \%)$ were in sinus rhythm on last follow-up and demonstrated maintenance sinus rhythm (MSR group). There were no significant differences with respect to gender, body mass index, AF type, AF 
Table 2 Univariate and multivariate logistic regression analysis of AF recurrence

\begin{tabular}{|c|c|c|c|c|}
\hline \multirow[b]{2}{*}{ Variables } & \multirow{2}{*}{$\begin{array}{l}\text { Univariable } \\
\text { OR }(95 \% \mathrm{Cl})\end{array}$} & \multirow[b]{2}{*}{$P$ value } & \multirow{2}{*}{$\begin{array}{l}\text { Multivariable } \\
\text { OR }(95 \% \mathrm{Cl})\end{array}$} & \multirow[b]{2}{*}{$P$ value } \\
\hline & & & & \\
\hline Age & 1.065 (1.005 to 1.128$)$ & 0.033 & 0.988 (0.886 to 1.068$)$ & 0.974 \\
\hline NT-pro-BNP & 1.001 (1.000 to 1.002$)$ & 0.003 & 0.881 (0.999 to 1.001$)$ & 0.906 \\
\hline AF type & 1.863 (0.865 to 4.015$)$ & 0.112 & 0.387 (0.102 to 1.392$)$ & 0.143 \\
\hline LVMI & 1.020 (0.997 to 1.043$)$ & 0.094 & 0.996 (0.961 to 1.021$)$ & 0.531 \\
\hline LAVImax & 1.047 (1.012 to 1.083$)$ & 0.008 & $1.025(0.977$ to 1.076$)$ & 0.311 \\
\hline LVEF, \% & 0.958 (0.900 to 1.019$)$ & 0.173 & $1.010(0.927$ to 1.101$)$ & 0.802 \\
\hline LAAFV & 0.933 (0.902 to 0.965$)$ & 0.000 & 0.940 (0.896 to 0.987$)$ & 0.011 \\
\hline GLS & 0.758 (0.680 to 0.845$)$ & 0.000 & 0.689 (0.528 to 0.772$)$ & 0.000 \\
\hline
\end{tabular}

AF, atrial fibrillation; GLS, global longitudinal strain; LAAFV, left atrial appendage emptying flow velocity; LAVImax, left atrial maximal volume index; LVEF, left ventricular ejection fraction; LVMI, left ventricular mass index; NT-pro-BNP, N-terminal pro-B-type natriuretic peptide; ;OR, odd ratio.

duration, common cardiovascular risk factors, current clinical medical treatment, sUA, TAPSE, LVMI and LVEF between the two groups at baseline. However, AFR group were older $(65.5 \pm 6.0$ vs . $62.6 \pm 7.3, \mathrm{p}=0.03)$, had higher NT-pro-BNP level $(1046.0 \pm 1044.2$ vs . 527.8 \pm 564.9 , $\mathrm{p}<0.001)$, larger LAVImax $(44.1 \pm 11.4$ vs $.37 .2 \pm 12.4$, $\mathrm{p}=0.001)$, lower LAAFV $(36.2 \pm 12.0$ vs . $50.3 \pm 16.9$, $\mathrm{p}<0.001)$ and lower GLS $(19.8 \pm 4.5$ vs $.26 .6 \pm 5.5, \mathrm{p}<0.001)$ when compared with MSR group. GLS showed a good intraobserver and interobserver variability, the intraobserver variation coefficient of GLS was $4.6 \% \pm 1.5 \%$ and the interobserver variation was $5.2 \% \pm 1.6 \%$.

\section{Development of predicted model and validation}

Univariate and multivariable predictors of AFR were shown in tables 2 and 3. Univariate logistic regression analysis identified that age (OR: $1.065,95 \%$ CI 1.005 to 1.128, $\mathrm{p}=0.033$ ), NT-pro-BNP (OR: 1.001, 95\% CI 1.000 to $1.002, \mathrm{p}=0.003$ ), LAVImax (OR: 1.047, 95\% CI 1.012 to $1.083, \mathrm{p}=0.008$ ), LAAFV (OR: $0.933,95 \%$ CI 0.902 to $0.965, \mathrm{p}<0.001)$ and GLS (OR: $0.758,95 \%$ CI 0.680 to $0.845, \mathrm{p}<0.001)$ were significant contributors to AFR after ablation and were included in the multivariate analysis. Multivariate logistic regression analysis found LAAFV (OR: $0.940,95 \%$ CI 0.896 to $0.987, \mathrm{p}=0.011$ ) and GLS (OR: $0.689,95 \%$ CI 0.528 to $0.772, \mathrm{p}<0.001$ ) were independent factors for prediction the possibility of AFR after ablation.

Then, the variables in multivariable logistic analysis with significant level $\leq 0.5$ were selected for development of prediction nomogram model, including AF type (OR: $0.394,95 \%$ CI 0.114 to $1.368, \mathrm{p}=0.142$ ), LAAFV (OR: $0.941,95 \%$ CI 0.899 to $0.984, \mathrm{p}=0.008$ ), LAVImax (OR: $0.643,95 \%$ CI 0.538 to $0.769, \mathrm{p}=0.253$ ) and GLS (OR:1.023, 95\% CI 0.984 to 1.064 , p $<0.001$ ). Bootstrapping with 1000 resamples was performed to quantify and predict future performance for internal validation. The C-index of the nomogram for prediction AFR was 0.901. The calibration plot demonstrated good agreement between prediction and actual recurrence (figures 2 and $3)$.

The most predictive variables entered comparative ROC analysis, which identified that the area under

Table 3 Multivariable logistic regression analysis of AF recurrence and nomogram construction

\begin{tabular}{|c|c|c|c|c|}
\hline \multirow[b]{2}{*}{ Variables } & \multirow{2}{*}{$\begin{array}{l}\text { Multivariable } \\
\text { OR }(95 \% \mathrm{Cl})\end{array}$} & \multirow[b]{2}{*}{$P$ value } & \multirow{2}{*}{$\begin{array}{l}\text { Selected for model } \\
\text { OR }(95 \% \mathrm{Cl})\end{array}$} & \multirow[b]{2}{*}{$P$ value } \\
\hline & & & & \\
\hline Age & $0.988(0.886$ to 1.068$)$ & 0.974 & & \\
\hline NT-pro-BNP & 0.881 (0.999 to 1.001$)$ & 0.906 & & \\
\hline AF type & 0.387 (0.102 to 1.392$)$ & 0.143 & 0.394 (0.114 to 1.368$)$ & 0.142 \\
\hline LVMI & 0.996 (0.961 to 1.021$)$ & 0.531 & & \\
\hline LAVImax & 1.025 (0.977 to 1.076$)$ & 0.311 & 0.643 (0.538 to 0.769$)$ & 0.253 \\
\hline LVEF, \% & 1.010 (0.927 to 1.101$)$ & 0.802 & & \\
\hline LAAFV & 0.940 (0.896 to 0.987$)$ & 0.014 & 0.941 (0.899 to 0.984) & 0.008 \\
\hline GLS & $0.689(0.528$ to 0.772$)$ & 0.000 & 1.023 (0.984 to 1.064$)$ & 0.000 \\
\hline
\end{tabular}

AF, atrial fibrillation; GLS, global longitudinal strain; LAAFV, left atrial appendage emptying flow velocity; LAVImax, left atrial maximal volume index; LVEF, left ventricular ejection fraction; LVMI, left ventricular mass index; NT-pro-BNP, N-terminal pro-B-type natriuretic peptide.; 
Points

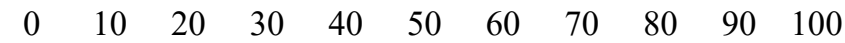

Persistent

AF type Paroxysmal

LAAFV

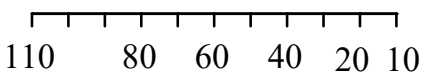

LAVImax

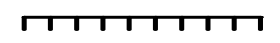

GLS $\begin{array}{llll}20 & 50 & 80 & 100\end{array}$

\begin{tabular}{lllllll}
\hline & 1 & 1 & 1 & \\
40 & 35 & 30 & 25 & 20 & 15 & 10
\end{tabular}

\section{AF Reccurence rate}

$\begin{array}{lllllllll}0.05 & 0.1 & 0.2 & 0.4 & 0.6 & 0.8 & 0.9 & 0.95\end{array}$

Figure 2 Nomogram for prediction patients with AF recurrence. It can be easily used to following steps: (1) affirm the AF type, the value of LAAFV, LAVImax and GLS; (2) Sum up all the points of each patient and locate the calculated total number on the total points axis; (3) The total points determine final probabilities of AF recurrence. AF, atrial fibrillation; GLS, global longitudinal strain; LAAFV, left atrial appendage emptying flow velocity; LAVImax, Left Atrial Maximal Volumes Index.

curve (AUC) of combined index (GLS+LAAFV + LAVImax+AF type) to be superior compared with lonely AF type, LAVImax, LAAFV and GLS for the prediction of AFR, suggesting that combined index has the highest diagnostic performance (AUC $0.880,95 \%$ CI 0.812 to 0.947 vs AUC $0.576,95 \%$ CI 0.470 to 0.683 , AUC 0.684 , $95 \%$ CI 0.585 to 0.784 , AUC $0.744,95 \%$ CI 0.654 to

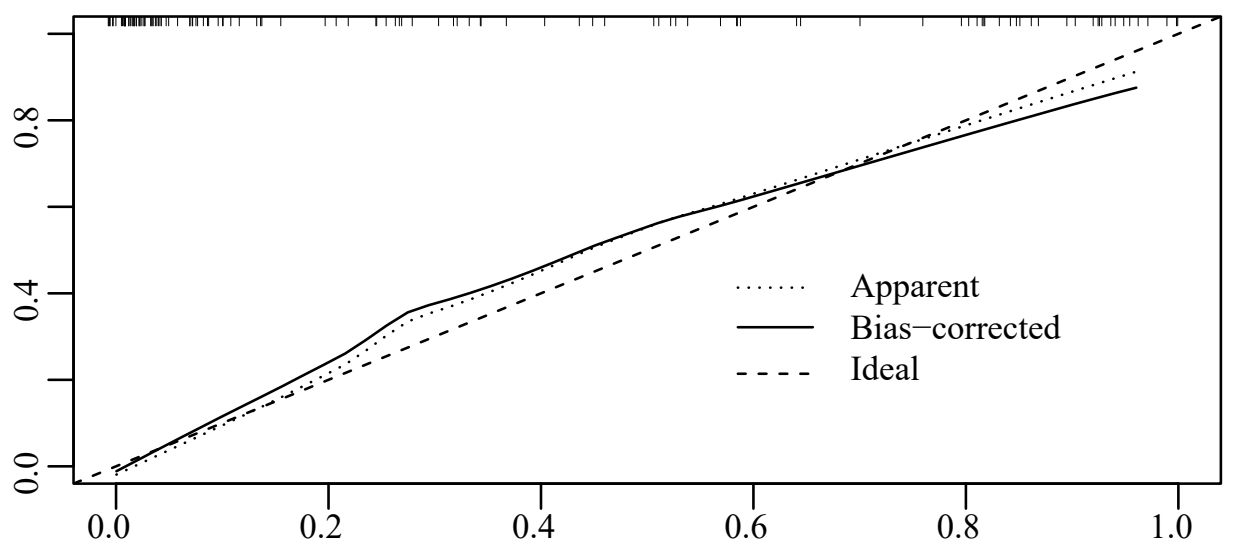

Predicted $\operatorname{Pr}\{$ Reccurence $=1\}$

$B=1000$ repetitions, boot

Mean absolute error $=0.031 \mathrm{n}=124$

Figure 3 The calibration curve was used to evaluate the prediction performance of AF recurrence after ablation therapy of the non-invasive nomogram with $\mathrm{C}$-index of 0.901 , indication a good performance. The short line represented an ideal evaluation, the dotted line represented apparent evaluation and the solid line stand for bias-corrected evaluation. AF, atrial fibrillation. 


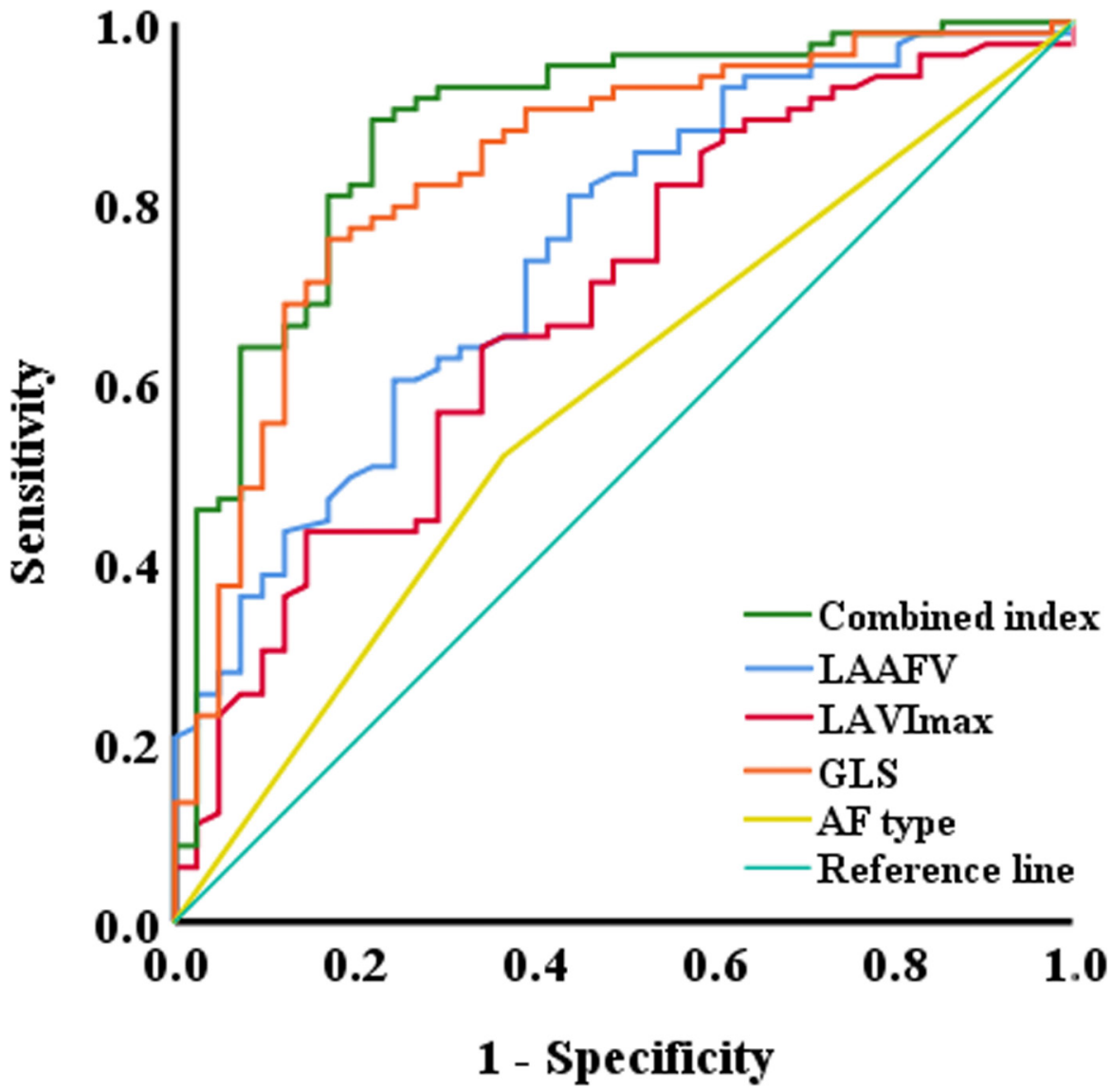

Figure 4 ROC curves for the prediction of sinus rhythm maintenance. The area under the curve (AUC) for combined index was greater than for left atrial global longitudinal strain (GLS), left atrial appendage emptying flow velocity (LAAFV), maximal LA Volume Index (LAVImax) and AF type. AF, atrial fibrillation.

0.834 , AUC $0.839,95 \%$ CI 0.470 to 0.683 , respectively) (figure 4).

\section{DISCUSSION}

This study developed an easy-to-use score system based on AF type, LAVImax, LAAFV and GLS for predict of AFR postablation. The major findings in the current study were as follows. First, AFR patients showed a significantly decreased GLS and LAAFV as compared with MSR group indicating a functional remodelling occur to LA in patients with AF. Second, GLS and LAAFV were independent predictor of AFR after ablation after adjustment for clinical characteristics and conventional echocardiographic parameters, which allows identification of patients with high risk for AFR after ablation. Third, an integrated scoring model that combined AF type, LAVImax, and LAAFV and GLS showed excellent discriminatory performance (C-index: 0.901).

With the development of non-invasive assessment techniques of LA function and LA fibrosis, LA function (such as LA volume, LVEF and LA strain) and LA fibrosis degree assessed by delayed enhancement MRI become good predictive factors of AFR in patients post-AF ablation. ${ }^{916-19}$ These studies were based on the same concept: LA structural and functional remodelling may be the possible pathogenesis of AFR following catheter ablation. ${ }^{20}$ Thus,
LA strain, a sensitive indicator of LA function, combined with LA volume, a structural parameter of LA, will help to predict the recurrence of AF after ablation. The nomogram we proposed in our study included the above two indicators and provided an easy-to-use predictive model to identify who had high possibility of AFR. Patients with an estimated value of more than 88 points with the AFR more than $50 \%$ in our model, so more reasonable decisions such as additional LAA isolation or intensive drug treatment and frequent follow-up should be considered for those patients.

The nomogram included four predictors from baseline clinical data, TEE, TTE and STE analysis prior to ablation. GLS and LAAFV were the important independent risk factors of AFR in the multivariate model. The nomogram established based on this multivariate regression model showed optimal discrimination (C-index 0.901), indicating a good performance for predict of rhythm outcome post-AF ablation. In this study, we attempted to evaluate the combination predictive value of AF type, LAVImax, LAAFV and GLS. Interestingly, the discriminant ability of the predictive model was greatly improved by adding GLS to the model involving clinical variables, TTE and TEE parameters.

Our analysis results were accordance with the previous findings. Notably, persistent AF has been reported as a 
significant predictor for higher rates of recurrent $\mathrm{AF}$, which may be due to a higher degree of atrial remodelling in patients with persistent AF. A long-term process of reverse remodelling initiated by ablation might be essential to restore and MSR. ${ }^{21}{ }^{22}$ Therefore, the inducibility of $\mathrm{AF}$ was also identified as a significant predictor of recurrent $\mathrm{AF}^{23}$ Current international guidelines suggested that LA volume should be instead of assessing the LA size and remodelling, particularly in patients with dilated atria, due to the asymmetrical nature of enlargement atria. ${ }^{24}$ Recent trials have demonstrated that increased LAVI was associated with occult AFR postablation. ${ }^{25}{ }^{26}$ In addition, the experimental evidence also implied that patients with decreased LAA function prior to ablation are more likely to relapse. ${ }^{27} \mathrm{LAAFV}$ reflects the comprehensive LA function and severity of LA remodelling and has been considered as a reliable predictor of AFR. ${ }^{28}$ GLS measured by STE for the assessment of LA reservoir function has showed its prognostic value in predicting the recurrent of $\mathrm{AF}$, as it provides characterisation and quantification of LA myocardial deformation, including the compliance of the atrial wall as well as myocardial fibrosis. ${ }^{29}{ }^{30}$ One might hypothesise that a lower GLS may mean a subclinical impairment of the LA myocardium, which can be linked to a higher risk of recurrence of $\mathrm{AF}$ post-AF ablation. Therefore, it is reasonable to include these important predictors in the nomogram.

When compared with the univariate and multivariate prediction models including clinical variables, echocardiographic parameters and strain values, this nomogram model showed better prediction accuracy. The findings of this study suggest that our nomogram scoring system can be used as a useful model for identifying the high AFR rate after catheter ablation, which is superior to the predictive value of a single parameter. Thus, the assessment of GLS and LAAFV may help clinicians to identify patients at relatively higher risk for recurrent of AF. Since the structural and functional remodelling of LA is partially reversible, the evaluation of GLS and LAAFV may provide more treatment recommendations for patients with $\mathrm{AF}$ ablation on the basis of drug therapy.

\section{Study limitations}

The present study has several main limitations. First, this is a single-centre study with a small sample size, so a prospective multicentre study needs to confirm the predictive value of GLS in predicting the rhythmic outcome of AF. Second, although patients were followed up through regular outpatient appointments, electrocardiograms and 24-hour Holter, asymptomatic episodes of AF may not be detected. Thus, the AFR rate may be underestimated in the study. In addition, obstructive sleep apnea (OSA) is a known risk factor for recurrent $\mathrm{AF}$, We will explore the effect of OSA on recurrence after ablation of AF in future studies. Finally, nomogram should be applied and validated in external cohorts with larger prospective studies. Therefore, more research is needed to test the validity of the nomogram we have established.

\section{CONCLUSION}

In conclusion, we have constructed an easy-to-use nomogram based on echocardiography features and clinical characteristics, which allowed physicians to identify patients who potentially benefit from catheter ablation, and provided practical guidance for individualised management of patients with $\mathrm{AF}$ after catheter ablation.

Contributors Image acquisition and strain analysis: X-XM and AW. Clinical data collection: KL. Analysis and interpretation of data: X-XM. All authors have given critical review of the article and approved the final version of the manuscript submitted.

Competing interests None declared.

Patient consent for publication Not required.

Ethics approval This study was approved by the institutional review boards of university of Shanghai Jiao Tong affiliated the sixth people's hospital and all patients provided written informed consent.

Provenance and peer review Not commissioned; externally peer reviewed.

Data availability statement Data are available on reasonable request.

Open access This is an open access article distributed in accordance with the Creative Commons Attribution Non Commercial (CC BY-NC 4.0) license, which permits others to distribute, remix, adapt, build upon this work non-commercially, and license their derivative works on different terms, provided the original work is properly cited, appropriate credit is given, any changes made indicated, and the use is non-commercial. See: http://creativecommons.org/licenses/by-nc/4.0/.

ORCID iD

Xin-Xin Ma http://orcid.org/0000-0003-2463-8108

\section{REFERENCES}

1 Calkins H, Hindricks G, Cappato R. HRS/EHRA/ECAS/APHRS/ SOLAECE expert consensus statement on catheter and surgical ablation of atrial fibrillation. Heart Rhythm 2017;2017:e275-444.

2 , Lloyd-Jones D, Adams RJ, et al, WRITING GROUP MEMBERS. Heart disease and stroke statistics--2010 update: a report from the American Heart Association. Circulation 2010;121:e46-215.

3 Bhatla A, Borovskiy Y, Katz R, et al. Stroke, timing of atrial fibrillation diagnosis, and risk of death. Neurology 2021;96:e1655-62.

$4 \mathrm{Kim} \mathrm{YG}$, Boo KY, Choi J-I, et al. Early recurrence is reliable predictor of late recurrence after radiofrequency catheter ablation of atrial fibrillation. JACC Clin Electrophysiol 2021;7:30979-8.

5 Chen S, Schmidt B, Bordignon S, et al. Catheter ablation of atrial fibrillation using ablation index-guided high-power technique: Frankfurt Al high-power 15-month follow-up. J Cardiovasc Electrophysiol 2021;32:616-24.

6 Park J-W, Yu HT, Kim T-H, et al. Mechanisms of long-term recurrence 3 years after catheter ablation of atrial fibrillation. JACC Clin Electrophysiol 2020;6:999-1007.

7 Okada M, Tanaka N, Tanaka K, et al. Usefulness of post-procedural plasma brain natriuretic peptide levels to predict recurrence after catheter ablation of atrial fibrillation in patients with left ventricular systolic dysfunction. Am J Cardiol 2021;144:057.

8 Toufan M, Kazemi B, Molazadeh N. The significance of the left atrial volume index in prediction of atrial fibrillation recurrence after electrical cardioversion. J Cardiovasc Thorac Res 2017;9:54-9.

9 Moreno-Ruiz LA, Madrid-Miller A, Martínez-Flores JE, et al. Left atrial longitudinal strain by speckle tracking as independent predictor of recurrence after electrical cardioversion in persistent and long standing persistent non-valvular atrial fibrillation. Int J Cardiovasc Imaging 2019;35:1587-96.

10 Mochizuki A, Yuda S, Fujito T, et al. Left atrial strain assessed by three-dimensional speckle tracking echocardiography predicts atrial fibrillation recurrence after catheter ablation in patients with paroxysmal atrial fibrillation. J Echocardiogr 2017;15:79-87.

11 Koca H, Demirtas AO, Kaypaklı O, et al. Decreased left atrial global longitudinal strain predicts the risk of atrial fibrillation recurrence after cryoablation in paroxysmal atrial fibrillation. $J$ Interv Card Electrophysiol 2020;58:51-9.

12 Hosseini K, Vasheghani-Farahani A, Hosseinsabet A. Decreased longitudinal systolic strain rate of the left atrial myocardium as one 
of the earliest markers of atrial cardiomyopathy in subjects with brief paroxysmal atrial fibrillation. J Clin Ultrasound 2020;48:476-85.

13 Lang RM, Badano LP, Mor-Avi V, et al. Recommendations for cardiac chamber quantification by echocardiography in adults: an update from the American Society of Echocardiography and the European Association of Cardiovascular Imaging. J Am Soc Echocardiogr 2015;28:e14:1-39.

14 Lee K-N, Choi J-I, Kim YG, et al. Comparison between linear and focal ablation of complex fractionated atrial electrograms in patients with non-paroxysmal atrial fibrillation: a prospective randomized trial. Europace 2019;21:598-606.

15 Wang Y, Xu Y, Ling Z, et al. Radiofrequency catheter ablation for paroxysmal atrial fibrillation: outcomes during a 3-year follow-up period. J Int Med Res 2019;47:1636-48.

16 Tan BYQ, Ho JSY, Sia C-H, et al. Left atrial volume index predicts new-onset atrial fibrillation and stroke recurrence in patients with embolic stroke of undetermined source. Cerebrovasc Dis 2020;49:285-91.

17 McGann C, Akoum N, Patel A, et al. Atrial fibrillation ablation outcome is predicted by left atrial remodeling on MRI. Circ Arrhythm Electrophysiol 2014;7:23-30.

18 Chou C-C, Lee H-L, Chang P-C, et al. Left atrial emptying fraction predicts recurrence of atrial fibrillation after radiofrequency catheter ablation. PLoS One 2018;13:e0191196.

19 Wang Y, Li M, Zhong L, et al. Left atrial strain as evaluated by two-dimensional speckle tracking predicts left atrial appendage dysfunction in Chinese patients with atrial fibrillation. Cardiol Res Pract 2020;2020:1-8.

20 Chelu MG, King JB, Kholmovski EG, et al. Atrial fibrosis by late gadolinium enhancement magnetic resonance imaging and catheter ablation of atrial fibrillation: 5-year follow-up data. J Am Heart Assoc 2018;7:e006313.

21 Pappone C, Oreto G, Rosanio S, et al. Atrial electroanatomic remodeling after circumferential radiofrequency pulmonary vein ablation: efficacy of an anatomic approach in a large cohort of patients with atrial fibrillation. Circulation 2001;104:2539-44.
22 Müller P, Schiedat F, Bialek A, et al. Total atrial conduction time assessed by tissue Doppler imaging (PA-TDI interval) to predict early recurrence of persistent atrial fibrillation after successful electrical cardioversion. J Cardiovasc Electrophysiol 2014;25:161-7.

23 Richter B, Gwechenberger M, Filzmoser P, et al. Is inducibility of atrial fibrillation after radio frequency ablation really a relevant prognostic factor? Eur Heart $J$ 2006;27:2553-9.

24 Harkness A, Ring L, Augustine DX, et al. Normal reference intervals for cardiac dimensions and function for use in echocardiographic practice: a guideline from the British Society of Echocardiography. Echo Res Pract 2020;7:X1.

25 Kim DY, Kim YG, Choi J-I, et al. A novel predictive model for late recurrence after catheter ablation for atrial fibrillation using left appendage volume measured by cardiac computed tomography. Int $J$ Cardiovasc Imaging 2021;10:1007.

26 Celik Al, Kanadasi M, Demir M, et al. Predictors of the paroxysmal atrial fibrillation recurrence following cryoballoon-based pulmonary vein isolation: assessment of left atrial volume, left atrial volume index, galectin-3 level and neutrophil-to-lymphocyte ratio. Indian Pacing Electrophysiol J 2019;19:9-14.

$27 \mathrm{Kim}$ YG, Min K, Hwang SH, et al. Blood flow volume of left atrial appendage measured by magnetic resonance imaging is improved after radiofrequency catheter ablation of atrial fibrillation. $J$ Cardiovasc Electrophysiol 2021;32:1111.

28 Kanda T, Masuda M, Sunaga A, et al. Low left atrial appendage flow velocity predicts recurrence of atrial fibrillation after catheter ablation of persistent atrial fibrillation. J Cardiol 2015;66:377-81.

29 Mouselimis D, Tsarouchas AS, Pagourelias ED, et al. Left atrial strain, intervendor variability, and atrial fibrillation recurrence after catheter ablation: a systematic review and meta-analysis. Hellenic J Cardiol 2020;61:154-64.

30 Bai Y, Zhao Y, Li J, et al. Association of peak atrial longitudinal strain with atrial fibrillation recurrence in patients with chronic lung diseases following radiofrequency ablation. Intern Med $J$ 2018;48:851-9. 